The Astrophysical Journal, 292:90-103, 1985 May 1

(C) 1985. The American Astronomical Society. All rights reserved. Printed in U.S.A.

\title{
NOVA SHELLS. II. CALIBRATION OF THE DISTANCE SCALE USING NOVAE
}

\author{
JUDITH G. COHEN \\ Palomar Observatory, California Institute of Technology \\ Received 1984 September 20; accepted 1984 November 15
}

\begin{abstract}
Eight new spatially resolved nova shells have been found by imaging with a digital detector through a narrow $\mathrm{H} \alpha$ filter, and two old novae have been recovered. The 11 novae with the best determined maximum luminosities at outburst of the sample of 21 novae with reliable distances are used to derive a $M_{V}(\max )$-rate of decline relationship. These 11 objects have a mean $M_{V} 15$ days past maximum of $-5.60 \pm 0.45$ mag. If I assume that $M_{V}(15)$ is in fact constant, as is suggested by the theory, then for the full sample of 21 objects I find $M_{V}(\max$, corr $)=-10.70( \pm 0.30)+2.41( \pm 0.23) \log \left(t_{2}\right)$, where $t_{2}$ is the time in days to decline $2 \mathrm{mag}$ below maximum light. Having removed all obvious sources of observational error, I deduce that the dispersion in nova outburst luminosity for a fixed rate of decline is 0.45 mag. Previously published surveys of novae in M31 are used to redetermine the distance to that galaxy.

Subject headings: galaxies: individual - stars: novae
\end{abstract}

\section{INTRODUCTION}

Classical novae at maximum light appear to obey a luminosity-rate of decline relationship, as summarized by Schmidt (1957) and by McLaughlin (1960) and reviewed in Paper I (Cohen and Rosenthal 1983). A calibration of this relationship for the galactic novae is required in order to make use of novae as extragalactic distance indicators. The most reliable distances for galactic novae are those derived from the angular expansion of the shells. Therefore I have conducted an extensive search for more spatially resolved nova shells at Palomar Observatory over the past two years. The imaging observations of the eight newly resolved objects are described in $\S$ II, where a list of those novae which were not detected as resolved is also given. Two novae were recovered during the course of this survey, and finding charts for these objects are presented.

In $\S$ III the spectroscopic observations of these objects are described. In several cases the two-dimensional nature of the detector also permits a determination of the shell size from the spectra themselves. I also discuss the anomalous spectrum of the remnant of CK Vul 1670. The next section gives the absolute magnitude-rate of decline relationship for the 20 spatially resolved nova shells now known. The theory of nova light curves developed by Shara $(1981 a, b)$ is also compared with the actual observed behavior of novae. In the final section I summarize the results and indicate future prospects for improvement of the galactic calibration.

\section{IMAGING OF NOVAE}

\section{a) Philosophy of the Search}

The obvious way to find nova shells is to examine photographs taken through a suitable filter of historical galactic novae whose locations are known, and then look for spatial structure larger than that of a point source. Since nova shells emit primarily the Balmer lines and the forbidden lines of $\mathrm{O}$ and other abundant elements, and may be substantially reddened, a narrow $\mathrm{H} \alpha$ filter is the best choice for a nova shell search. The narrower the filter, the more the sky and the continuum from the postnova star are suppressed, so that a fainter shell can be detected. However, a filter which is too narrow compared with the width of the shell emission lines (typically of order $2000 \mathrm{~km} \mathrm{~s}^{-1}$ ) will suppress emission from parts of the shell and complicate determination of the shell size.

The selection of objects to be imaged was the collection of classical novae with outbursts prior to 1976 which were brighter than $V=6.6 \mathrm{mag}$ at maximum light and accessible from Palomar. The compilations of Payne-Gaposhkin (1957, 1977) were used to produce a sample of objects. Novae more recent than 1976 have shells that have not yet expanded enough to be detectable from the ground. Older novae with fainter maxima are unlikely to yield positive results, since they are probably too distant and their shells are now too faint. For many of the novae with outbursts prior to 1950, accurate positions and finding charts do not exist; such objects must first be recovered by imaging and confirmed spectroscopically.

A digital two-dimensional detector with the highest possible efficiency and lowest possible noise is required to detect these faint shells. In the 1983 observing season, the PFUEI (Gunn and Westphal 1981) was used at the prime focus of the $5 \mathrm{~m}$ Hale telescope, while in the 1984 season, the superb 4-shooter (Gunn et al. 1984) was used at the Cassegrain focus of the Hale telescope. The scale of the 4-shooter is $0.33 \mathrm{pixel}^{-1}$, while that of the PFUEI is $0.42 \mathrm{pixel}^{-1}$. The fast response of the automatic guider on the 4-shooter guarantees that image quality will be limited only by the atmosphere itself. Observations were generally carried out in dark time to suppress the sky as much as possible. Two filters were used: a filter whose FWHM is $30 \AA$ centered at $6570 \AA$ was used with PFUEI, while an even narrower filter $(16 \AA \mathrm{FWHM})$ centered at $\mathrm{H} \alpha$ was used with the 4-shooter. Typical exposure times were 30 minutes when the postnova star was suitably faint. To avoid saturation effects, when the postnova star was brighter $(r<16 \mathrm{mag})$, multiple shorter exposures were added.

\section{b) Nondetections and Nonrecoveries}

The nova shells proved to be faint and elusive. I first list in Table 1 those objects which were not found (in most cases after repeated efforts) to be spatially resolved. I give the $V$ magnitude at maximum light, and the current epoch $r$ magnitude in the Thuan-Gunn (1976) system and through the $16 \AA$ filter. (If the magnitudes are not given, the object was 
TABLE 1

Shells Not Detected

\begin{tabular}{|c|c|c|c|c|c|}
\hline Object & $\begin{array}{c}\text { Seeing } \\
\text { (FWHM) } \\
(\operatorname{arcsec})\end{array}$ & $\begin{array}{l}\text { Central Pixel } \\
\text { (DN) }\end{array}$ & $\begin{array}{c}m_{V}(\max )^{\mathrm{a}} \\
(\mathrm{mag})\end{array}$ & $\begin{array}{c}r \\
\text { (mag) }\end{array}$ & $\begin{array}{c}\mathrm{H} \alpha \\
(\mathrm{mag})\end{array}$ \\
\hline IV Cep 1971 . & 1.3 & 13,200 & 7.5 & 16.08 & 16.14 \\
\hline Q Cyg $1876 \ldots \ldots \ldots \ldots$ & 1.0 & 30,700 & 3.0 & 14.93 & 14.94 \\
\hline v450 Cyg $1942 \ldots \ldots \ldots$ & 1.0 & $5,600^{b}$ & 7.8 & 16.48 & 16.58 \\
\hline DI Lac $1910 \ldots \ldots \ldots \ldots$ & 1.0 & $58,675^{c}$ & 4.6 & 14.58 & 14.90 \\
\hline HR Lyr $1919 \ldots$. & 1.0 & 18,300 & 6.5 & 15.95 & 15.99 \\
\hline v841 Oph $1848 \ldots \ldots \ldots$ & 2.5 & 11,700 & 5.0: & $\ldots$ & $\cdots$ \\
\hline v368 Sct $1970 \ldots \ldots \ldots \ldots$ & 1.0 & 9,070 & 7.0 & $\cdots$ & 12.71 \\
\hline v373 Sct $1975 \ldots \ldots \ldots$ & 1.0 & $3,600^{b}$ & 6.0 & 18.07 & 16.98 \\
\hline WY Sge $1783 \ldots \ldots \ldots$ & 1.0 & 4,120 & 6: & 18.10 & 17.89 \\
\hline
\end{tabular}

a From Payne-Gaposchkin 1957, 1977.

b Faint star(s) closer than 4" to postnova star.

c Central pixel saturated. Its value was extrapolated using the point-source image profile.

observed through thin cirrus.) The $\mathrm{H} \alpha$ magnitude is normalized so that the magnitude of the standard stars was the same as in the $r$-filter. A correction of $0.23 \mathrm{mag}$ was then added to account for the effect of $\mathrm{H} \alpha$ absorption in the standard stars (usually subdwarfs). This correction factor was determined from high-dispersion spectra of a few standards, but undoubtedly varies somewhat depending on the set of standards observed on any given night. However, a difference in magnitude between $r$ and $\mathrm{H} \alpha$ of approximately 0.0 mag represents an object with a continuous spectrum in the region near $6550 \AA$. For each object I list the FWHM in seconds of arc of stellar images near the historical nova on the best frame I have obtained. I also give the detected data number (DN; the CCD detector electronics are adjusted so that $1 \mathrm{DN}$ is 2 electrons) in the central pixel of the nova image. Note that an excess of 50 $\mathrm{DN}$ in a pixel is easily seen, since the readout noise is less than 10 electrons (5 DN) and the sky, even on 30 minute exposures, is less than $400 \mathrm{DN}$. Thus, for example, Q Cyg 1876 was not seen to be extended to a limiting signal level of $0.16 \%$ of that of the central pixel of the star, or $0.03 \%$ of the stellar brightness (with a total of $164 \times 10^{3} \mathrm{DN}$ over the entire stellar image) through the $16 \AA$ wide filter. Because the size of the shell is unkown, it is not possible to convert these upper limits in shell surface brightness to a total brightness of the shell.

It would be useful to repeat the search for shells around the three novae of the early 1970s in another 5 years (or from space), since they show strong $\mathrm{H} \alpha$ emission but have not yet expanded enough to be resolved from the ground.

In spite of extensive literature searches, I could find only inadequate charts for several of the older novae. M. Liller kindly provided prints of the highest scale plates available near maximum light in the Harvard collection for several of the novae. I then determined magnitudes from my 4-shooter frames in $r$ and in $\mathrm{H} \alpha$ for the stars near the nova position, in the hope that an object whose magnitude difference indicated emission could be found. Two novae were recovered in this way, XX Tau 1927 and v500 Aql 1943. In spite of a careful search, EL Aql 1927, v630 Sgr 1936, and v840 Oph 1917 could not be recovered. (If any reader knows where they are, please write to me.)

\section{c) Detected Shells}

Eight new spatially resolved nova shells were found in the course of the past two years. Five objects (v500 Aql 1943, v1500 Cyg 1975, v446 Her 1960, XX Tau 1927, and LV Vul 1968) were well resolved, while three were marginally resolved (Aql 1970, DK Lac 1950, and RW UMi 1956), but each of these was detected as resolved on more than one exposure. Photographs of some of the shells are shown in Figure 1, which also provides a finding chart for the two newly recovered novae. The high accuracy of these digital images permits determination of the position of the centroid of a circularly symmetric image with an uncertainty of less than 0.1 pixels. Once the centroid is determined, I calculate radial image profiles using the distance of the center of each pixel from the image centroid. Examples of such profiles are shown in Figure 2. These raw profiles are then averaged over 1 pixel intervals to produce the final radial profiles. If nearby stars impinge on the image of the nova, as is shown in the case of LV Vul 1968 in Figure 2, the lower envelope of the distribution is used to define the radial profile. Radial profiles for each of the newly resolved novae (filled symbols) and the mean radial profile of a point source on the same frame (open symbols) determined from the mean of the profiles for five nearby stars of comparable or greater brightness than the nova through the $16 \AA$ filter are given in Figures $3 a-3 c$. (A profile of v533 Her 1963 is also included, since the profile given in Paper I came from an image with very poor spatial resolution.)

Several considerations enter in deriving a shell size from the observational data. All the detected shells appear circular, so I assume spherical symmetry. Models with varying ratios of shell brightness to point-source brightness were convolved with the seeing profile as determined by nearby stars, and the parameters adjusted until a best fit was obtained. Two parameters affect the shell profile, the radius of the shell and the extent to which the entire spherical volume is filled. Since tests to be described below show that the latter is not of major importance, I initially assume the profile of filled spherical shells. The $\mathrm{H} \alpha$ filter has a full width at half-maximum of $16 \AA$, so a shell expanding with a velocity of more than $360 \mathrm{~km} \mathrm{~s}^{-1}$ will have a distorted spatial profile. The filter transmission was approximated by a triangle, and it was assumed that the shell emits only in $\mathrm{H} \alpha$, not in [N II] $\lambda 6584$ or $\lambda 6548$, to derive the intrinsic shell radial profile. The expansion velocities used in the fitting procedure are those described in $\S$ III. The best fitting radial profile for each of the newly discovered shells is indicated in Figure 3 as a solid line, while the derived shell radii are listed in Table 2.

One may ask how unique the fit is, and to what extent the shell radii are compromised by our assumptions of spherical 


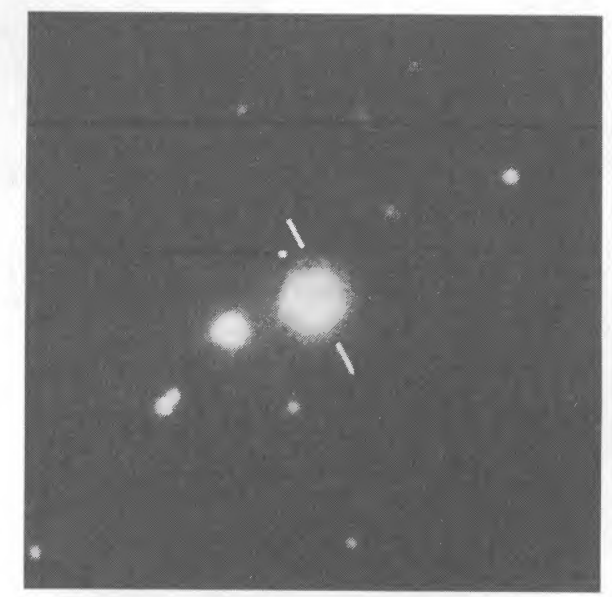

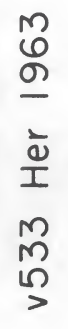
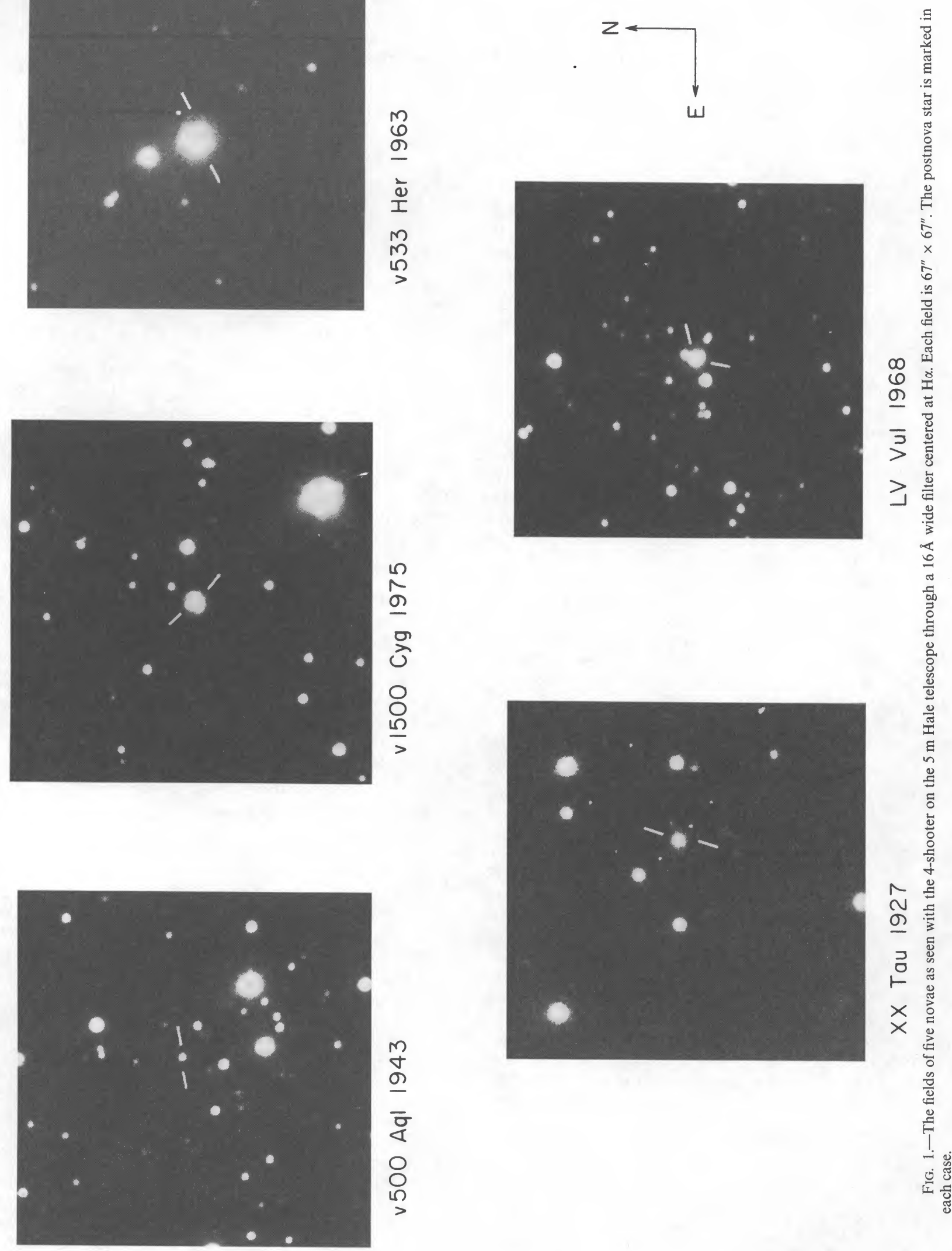

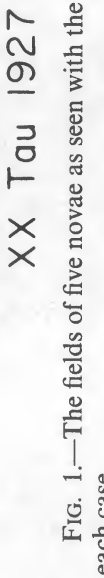



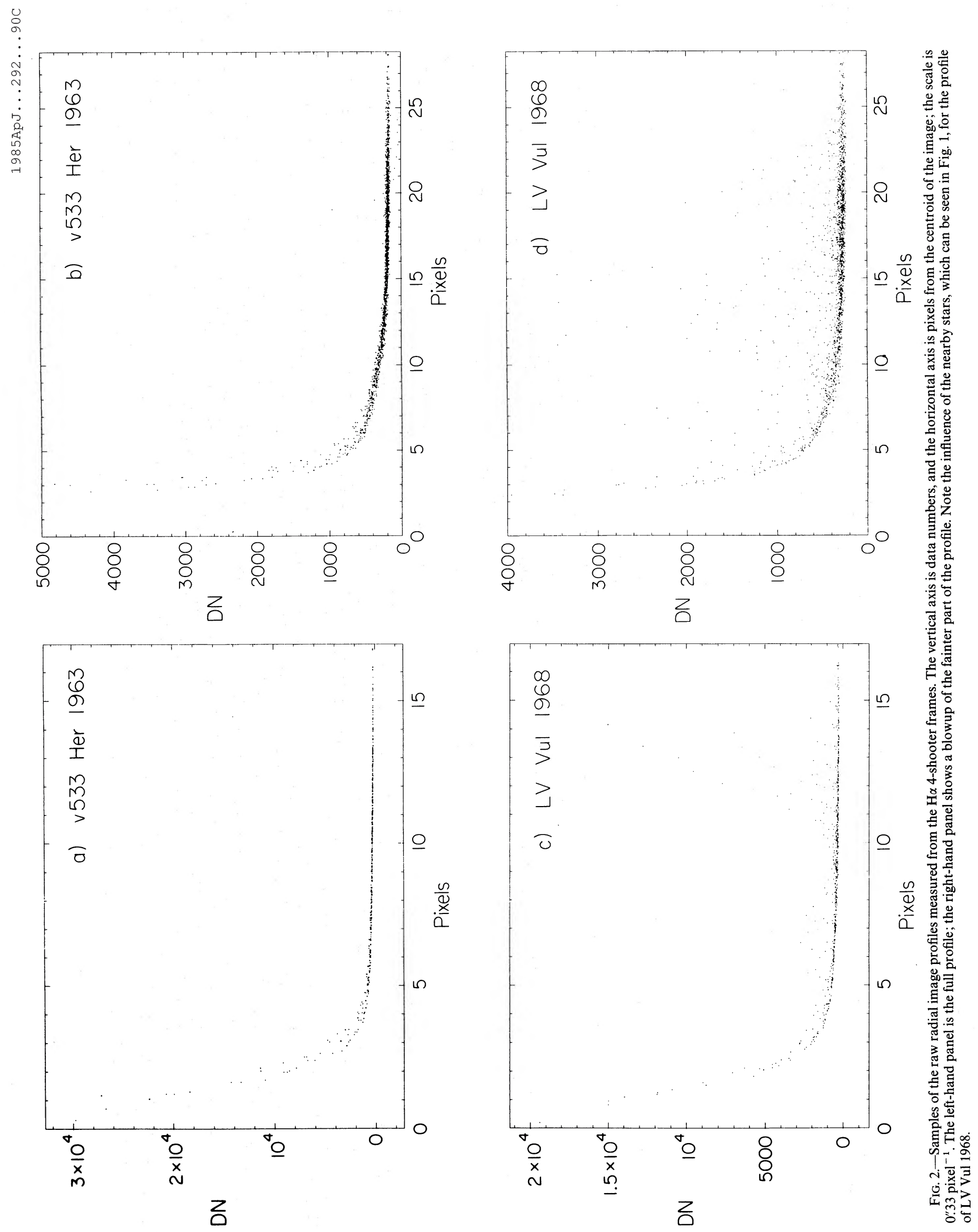

93 

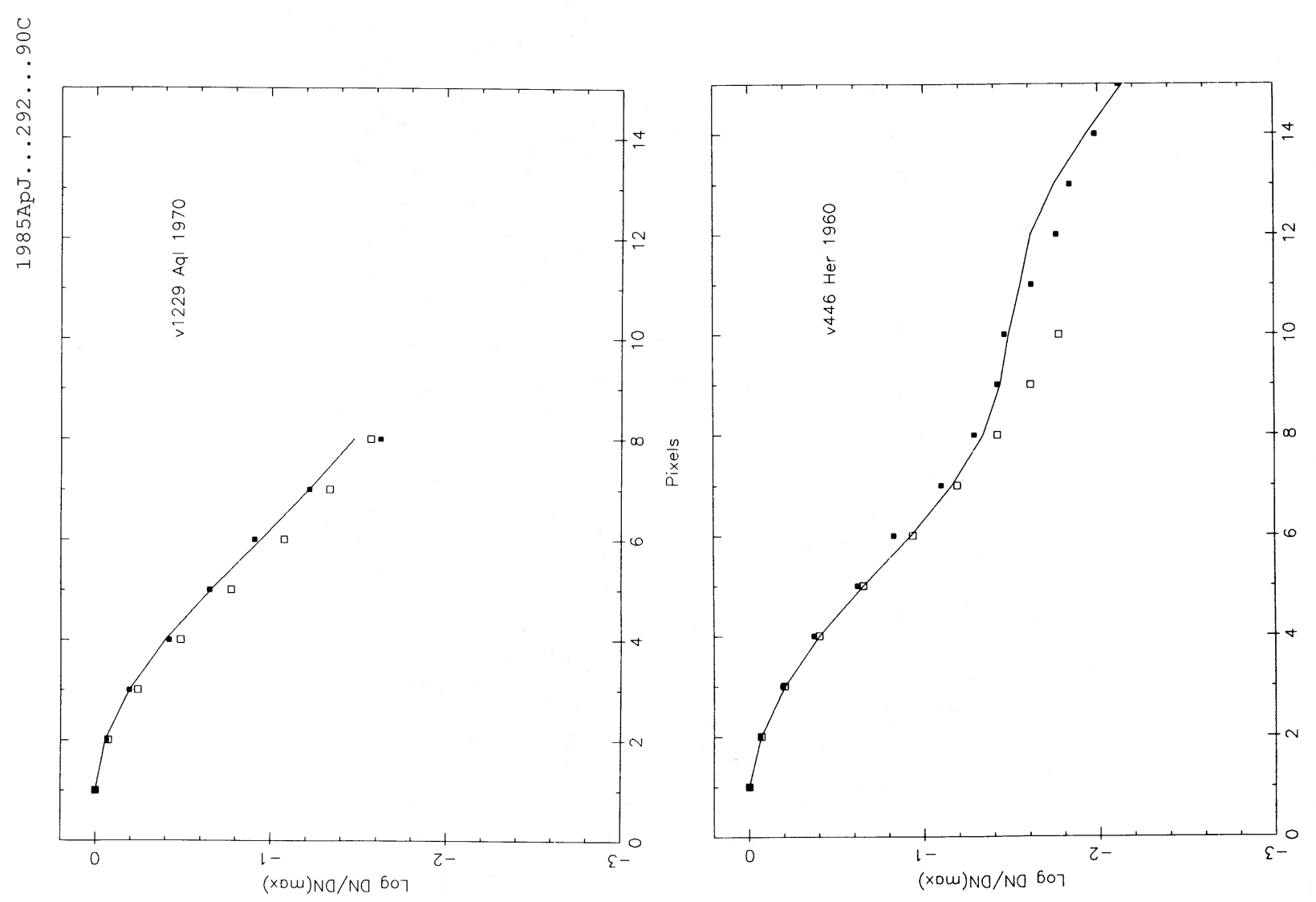

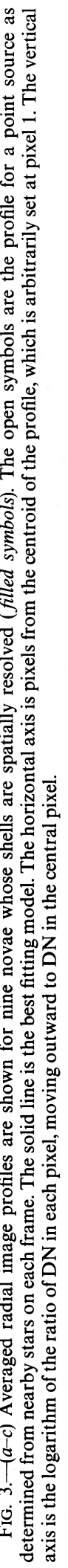
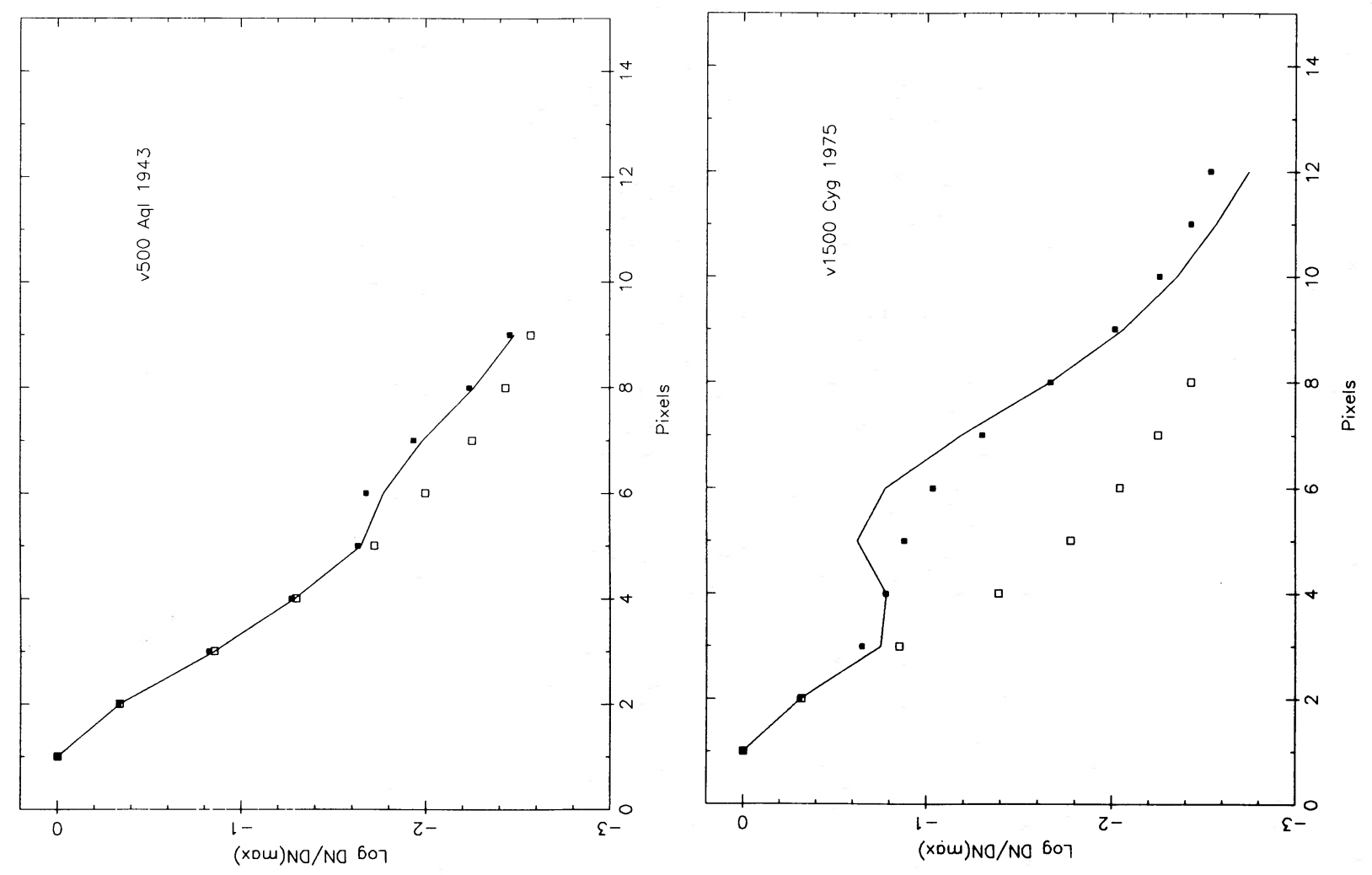

กั

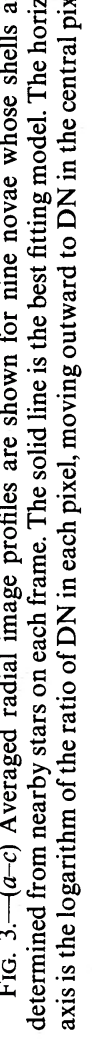



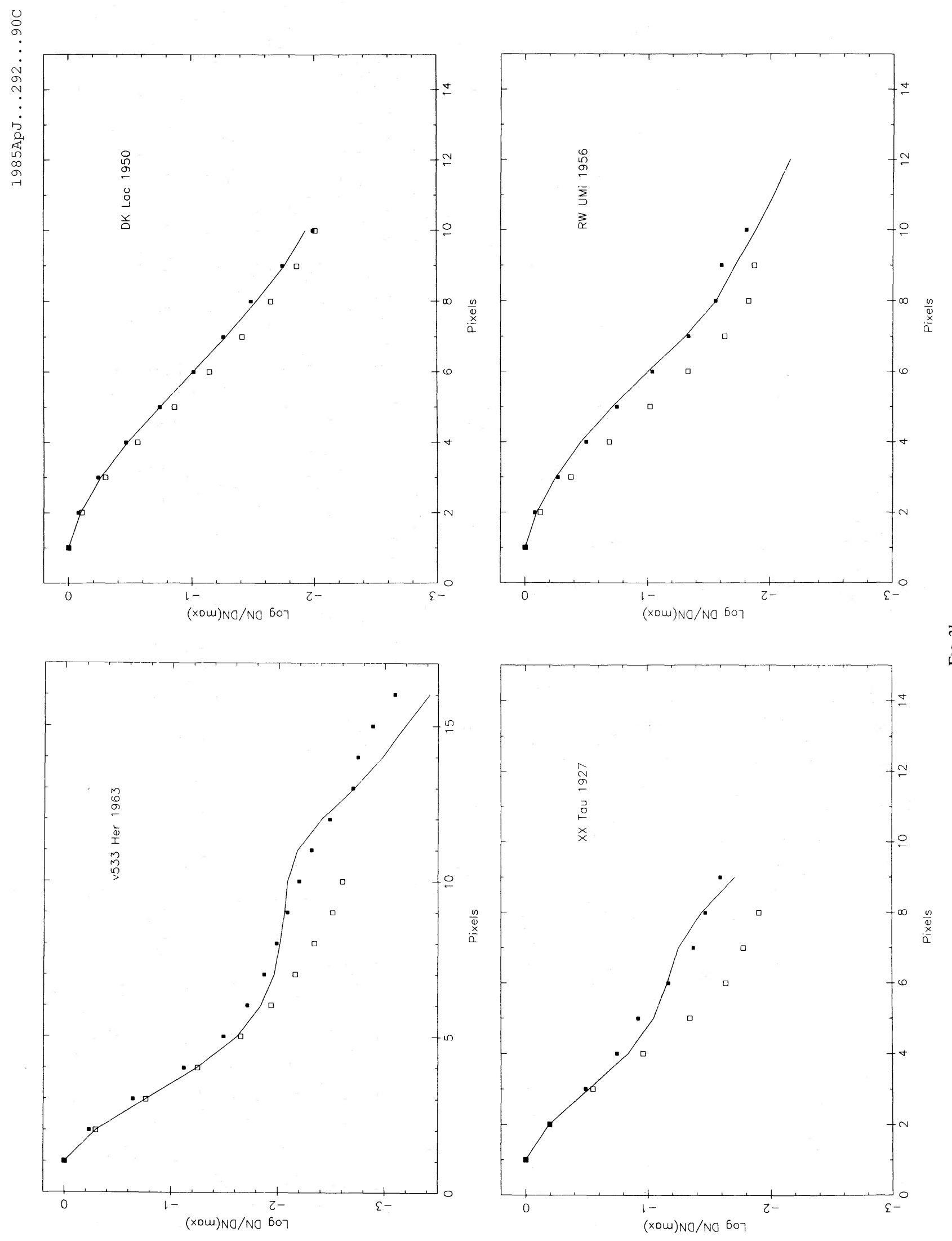

95

(C) American Astronomical Society - Provided by the NASA Astrophysics Data System 


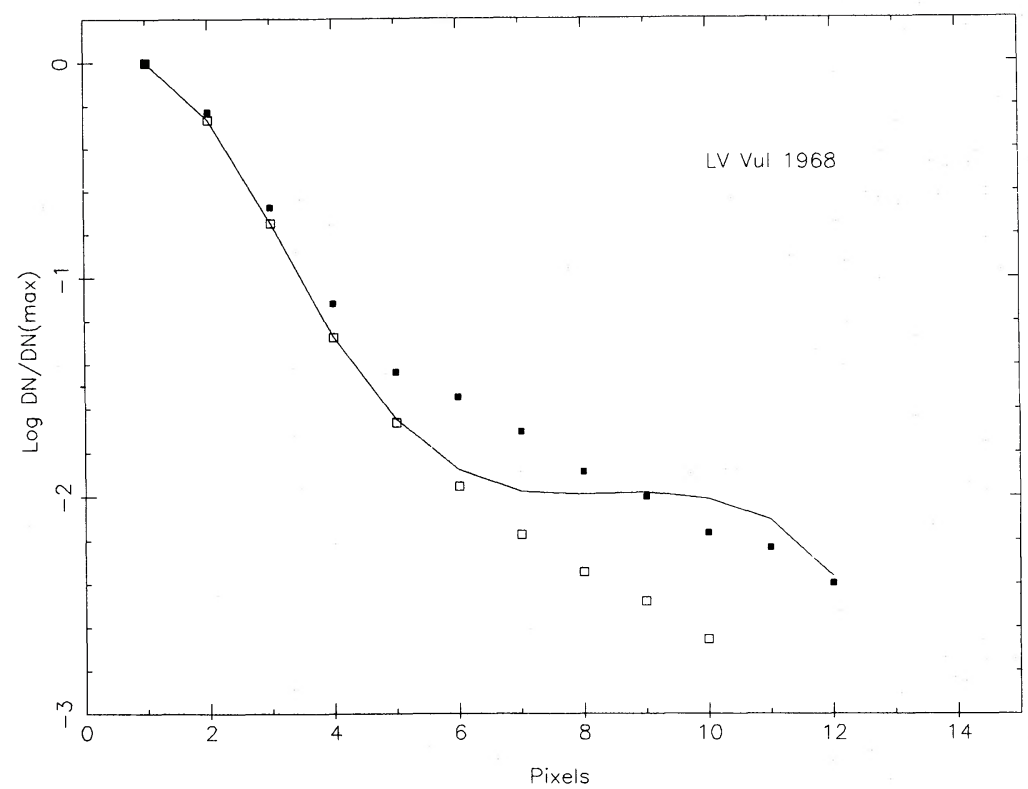

Fig. $3 c$

symmetry, no [N II] emission, and completely filled shells. Because of the high accuracy of the radial profiles, the best fit for a given theoretical profile and point-source spread function is well determined. As a measure of the quality of the fit, we use the parameter $s$ defined as

$$
s=\sum_{x=1}^{n}\left|\log \left[\frac{p(x)}{f(x)}\right]\right|,
$$

where the nova radial profile, $p(x)$, is determined over the interval in $x$ from the centroid of the image $(x=1)$ to $n$ pixels from the center of the image, and $f(x)$ represents the theoretical profile convolved with the seeing function. In this sum, points where the observed profile is less than $0.1 \%$ of the maximum are given half-weight because of their larger uncertainty. Figure 4 presents a plot of contour levels of the fit-quality parameter $s$ as a function of shell size and brightness ratio between the central point source and the shell for the rather small shell of v500 Aql 1943. The quality of the fit deteriorates noticeably when the radius is altered by more than 1 pixel $(0.33)$ from the best fit. Good discrimination of shell radius also occurs for larger shells.

The assumption of circular symmetry is justified by the visual appearance of the shells in the frames and by their radial profiles. All the newly discovered shells appear circular both in

TABLE 2

SPatially Resolved Novae

\begin{tabular}{|c|c|c|c|c|c|}
\hline \multirow[b]{2}{*}{ ОвJеCт } & \multicolumn{2}{|c|}{ SHELL RADIUS (ARCSEC) } & \multirow[b]{2}{*}{ ADOPTED } & \multirow{2}{*}{$\underset{\text { (mag) }}{r}$} & \multirow{2}{*}{$\begin{array}{c}\mathrm{H} \alpha \\
(\mathrm{mag})\end{array}$} \\
\hline & Imaging & Spectra & & & \\
\hline v500 Aql 1943 & 2.0 & & 2.0 & 19.32 & 19.09 \\
\hline v1229 Aql $1970 \ldots \ldots \ldots$ & 0.7 & $<1.5$ & 0.7 & $\ldots$ & $\ldots$ \\
\hline v1500 Cyg $1975 \ldots \ldots$. & 1.7 & $\ldots$ & 1.7 & 16.49 & 15.99 \\
\hline v446 Her $1960 \ldots \ldots \ldots$ & 5.0 & 4.2 & 4.5 & $\ldots$ & $\ldots$ \\
\hline v533 Her $1963 \ldots \ldots \ldots$ & 3.7 & 3.4 & 3.5 & 16.39 & 14.74 \\
\hline DK Lac $1950 \ldots \ldots \ldots$ & 2.0 & 2.0 & 2.0 & $\ldots$ & $\ldots$ \\
\hline XX Tau 1927 . & 2.3 & 2.0 & 2.2 & 19.45 & 18.94 \\
\hline RW UMi $1956 \ldots \ldots \ldots$ & 1.0 & $<1.5$ & 1.0 & 19.02 & 18.55 \\
\hline LV Vul $1968 \ldots \ldots \ldots \ldots$ & 3.7 & 2.0 & 2.8 & 15.37 & 15.39 \\
\hline
\end{tabular}

the original digital frames when examined at a magnified scale and in the radial profiles.

The use of a theoretical radial profile for a partially filled shell decreases the contribution of the shell near the center of the image and increases it farther away from the point source representing the postnova star. This has the same effect as the filter cutoff, where the center of the shell, which has the largest radial velocity (either positive or negative), is strongly suppressed by the filter, while the edge of the shell, with zero radial velocity, is unaffected. Tests with various theoretical profiles corresponding to shells which are filled only over the outermost $20 \%$ of the shell radius indicate that the effect is small; an overestimate in radius by 1 pixel $(0$ ". 3$)$ typically occurs when a filled-shell profile is used to match the observed radial image profiles instead of a largely empty shell. This occurs in part because the inner part of the profile is always dominated by the point source representing the postnova star; thus changing the filling factor significantly affects the shell brightness, but not the shell radius.

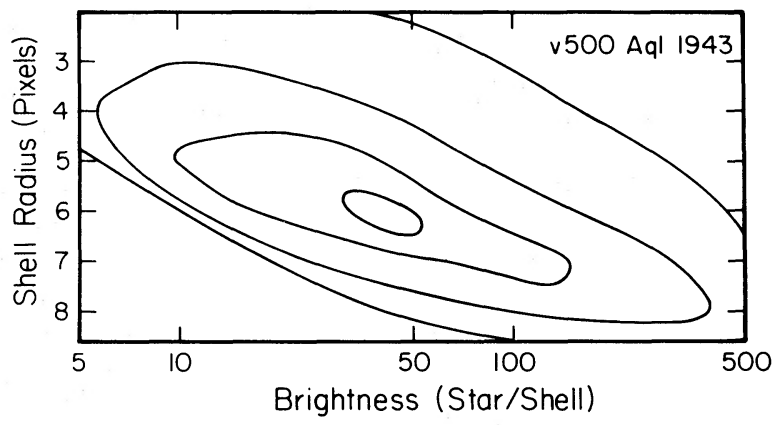

FIG. 4.-Contour levels of the quality-of-fit parameter $s$ defined in the text are plotted as a function of shell radius and of the brightness ratio of the postnova star to the shell. The case illustrated is the match of the observed radial image profile of v500 Aql 1943 to the theoretically calculated profiles. The contour levels shown are $s=0.04$ (the innermost contour), $0.07,0.10$, and 0.13 (the outermost contour, which is only partially within the boundaries of the figure). 

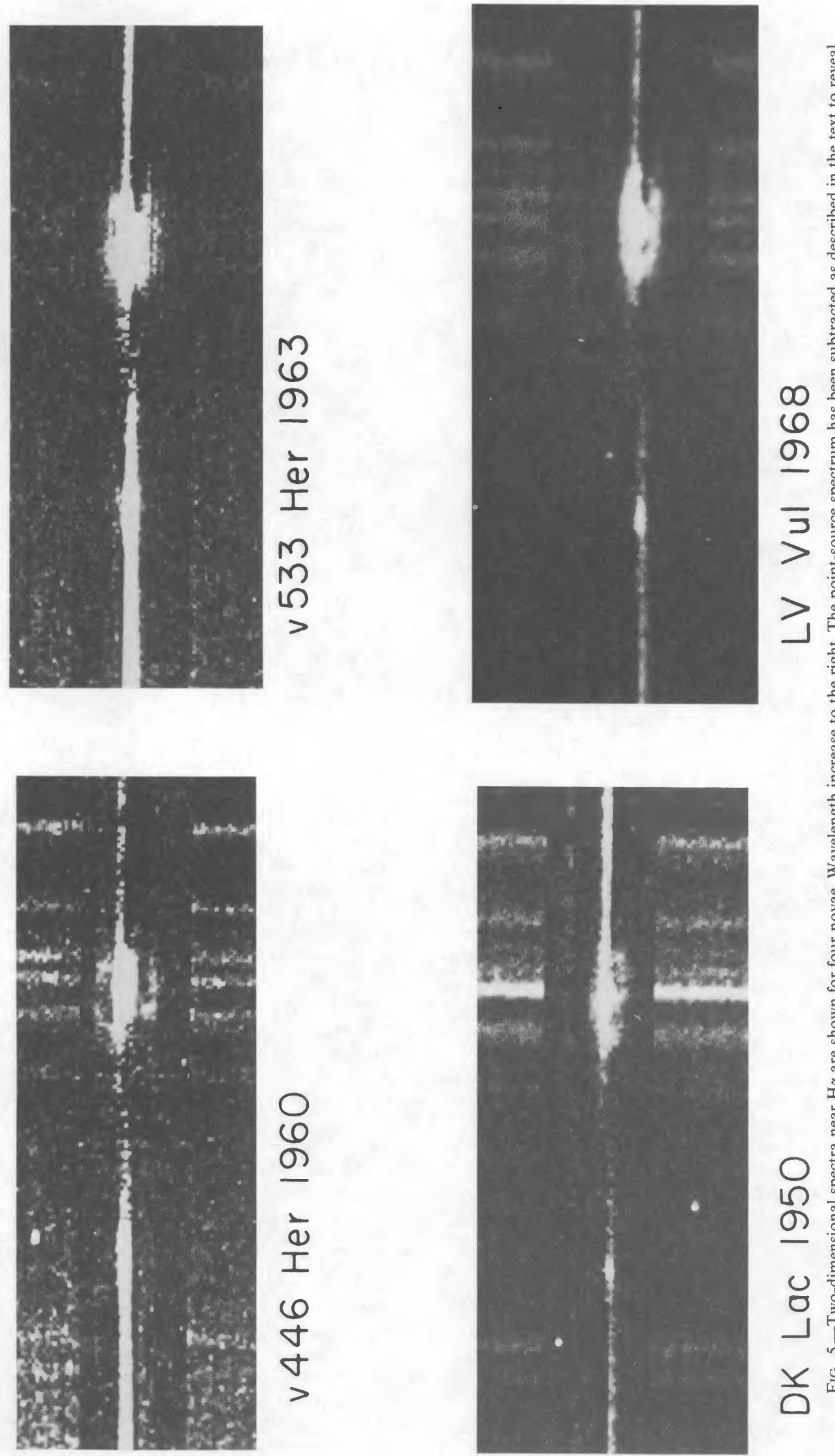

6

\%

$\overline{3}$

$>$

$>$

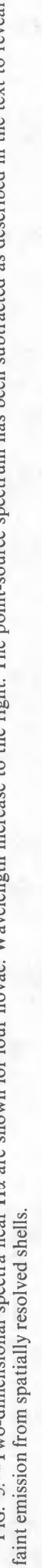


It is likely that the filter function used is in fact suppressing too severely the emission near the center of the shell, because in the younger shells the assumption that there is no [N $\mathrm{II}]$ emission is probably incorrect. The spectra discussed in $\S$ III indicate that in the older novae (with the exception of CK Vul 1670), $\mathrm{H} \alpha$ emission is much stronger than any [ $\mathrm{N} \mathrm{II}]$ emission.

In summary, the radii of the newly discovered shells listed in Table 2 have an maximum error of $+0.7,-0.3$.

\section{SPECTROSCOPIC OBSERVATIONS}

\section{a) The Expansion Velocities}

Spectroscopic observations were carried out for purposes of confirming the identifications of the newly recovered novae, measuring the expansion velocities of the shells, and obtaining the strengths of the interstellar lines toward the novae so as to deduce the interstellar absorption. I discuss here only the results for the novae whose shells have been spatially resolved and for one other nova of interest (CK Vul 1670). All the spectroscopic data were obtained with the Double Spectrograph described by Oke and Gunn (1982) at the Cassegrain focus of the $5 \mathrm{~m}$ Hale telescope at Palomar Mountain. The spectra of 1984 August 21 and 22 are particularly useful, as the seeing was extremely good $\left(1^{\prime \prime}\right)$, and high-wavelengthresolution gratings were used, yielding $1.6 \AA$ pixel $^{-1}$ in the red camera's CCD detector and $0.9 \AA$ pixel $^{-1}$ in the blue camera's two-dimensional photon counting detector (an improved version of that described by Shectman 1978). Figure 5 shows the region around $\mathrm{H} \alpha$ for several spectra. The data are shown in their original two-dimensional form. They have been flattened, and the sky spectrum was subtracted over a region approximately 30 pixels high centered on the source. Then a region of the spectrum $20 \AA$ wide near $6620 \AA$ was averaged perpendicular to the dispersion to obtain the profile of a point source, since there are no emission lines in that wavelength bandpass. This was subtracted from the original spectrum, to leave the spectrum of the shell only near $H \alpha$. (The residual continum shortward of $\mathrm{H} \alpha$ is due to the increasing signal level from the postnova star, so that the subtraction removes progressively less of the star.) These images were examined for evidence of the presence of a shell, and in several cases spatially resolved shell-like emission at $\mathrm{H} \alpha$ was detected, as shown in Figure 5. The radii of the shells were measured when possible and are listed in Table 2. (The scale of the red camera is 0 ".55 pixel $^{-1}$ perpendicular to the dispersion.)

Figure 5 demonstrates that in many cases the largest part of the $\mathrm{H} \alpha$ emission comes from a point source, presumably the accretion disk, while the shell itself contributes only a small fraction of the total $\mathrm{H} \alpha$ emission. The $\mathrm{He}$ I emission at $6678 \AA$ is also largely from the accretion disk, and there is no trace of [S II] in any of the spectra. Thus expansion velocities measured after a nova has faded more than 8 mag below maximum light (so that the shell emission lines no longer dominate the spectrum) may in fact be measures of the velocity gradients within the accretion disk unless the shell emission can be spatially resolved. This appears to have occurred in several cases among the novae studied in Paper I. For example, the expansion velocity of v533 Her 1963 from Paper I is approximately $500 \mathrm{~km} \mathrm{~s}^{-1}$ less than that measured in 1963 by Baschek (1964). The expansion velocity of LV Vul 1968 given in Paper I is 150 $\mathrm{km} \mathrm{s}^{-1}$ less than that of Hutchings (1970). Having realized this problem, I made a considerable effort to obtain expansion velocities from the literature as determined from spectra taken near maximum light (but not so close to maximum light that the various absorption systems and other such complications are still present). For most of the novae in Table 2, suitable expansion velocities could be found in the literature; the sources and expansion velocities adopted are listed in Table 3. The plate vaults of the Mount Wilson and Las Campanas Observatories yielded a rich collection of spectra of v446 Her 1960 (taken 1960 March 9-15), which I used to measure its expansion velocity. It was only necessary to adopt expansion velocities determined from $\mathrm{H} \alpha$ profiles as observed with the Double Spectrograph for two novae, RW UMi 1956 and XX Tau 1927. It is important for future research in this area that expansion velocities of new novae be measured while they are still bright enough that there is no uncertainty about the relative contributions of the shell and the accretion disk.

A similar plea arises for interstellar lines. It is not a trivial matter to measure to high accuracy the strength of interstellar lines in a postnova star which is now fainter than 18th magnitude. The equivalent widths of those interstellar lines which could be reliably detected are listed in Table 4.

\section{b) CK Vul 1670}

The nova CK Vul 1670 was recovered by Shara and Moffat (1982). A spectrum of part of the filamentary structure taken through a 4 " slit is shown in Figure 6. The slit orientation is indicated on the figure. The spectrum has been flattened and sky-subtracted, but not processed to remove a point source, unlike the spectra of Figure 5. A picture of CK Vul 1670 through an $r$-filter and through a $30 \AA$ wide $\mathrm{H} \alpha+[\mathrm{N}$ II $]$ filter taken with the PFUEI at the prime focus of the $5 \mathrm{~m}$ Hale telescope is also shown in Figure 6. These images show clearly that the shell is roughly circular, with bright filaments projecting outward beyond the radius of the fainter circular shell. The postnova star is not the object suspected by Shara and Moffat, which is in fact part of the shell, but may be the much fainter stellar object in the center of the shell (see also Shara, Moffat, and Webbink 1984). The spectrum of the shell of CK Vul 1670 is most peculiar, with strong [S II] emission (at 6717 and 6731 $\AA$ ) and [N II] emission (at 6584 and $6548 \AA$ ) and relatively weak $\mathrm{H} \alpha$ emission. The spectrum is closer to that of a supernova remnant; the ratios of line strengths $(3: 4: 14: 8: 5$ for 6731 , $6717,6584,6562$, and $6548 \AA$, respectively) in CK Vul 1670 agree well with the ratios compiled by Binnette et al. (1982) for galactic supernova remnants located inside the solar circle. However, the low expansion velocity and small size of the shell (approximately $10^{\prime \prime}$ in diameter) force us to accept CK Vul 1670 as a slow nova, rather than a supernova.

I estimate the time for a slow nova to sweep up interstellar material of the same mass as the ejected shell assuming no deceleration. Only one galactic nova studied here or in Paper I has an expansion velocity under $400 \mathrm{~km} \mathrm{~s}^{-1}$, so I adopt this as a minimum expansion velocity. The average interstellar $\mathrm{H}_{\mathrm{I}}$ density, which is adopted for the vicinity of the nova, is 0.4 $\mathrm{cm}^{-3}$ (see Shull and van Steenberg 1984). This does not include the local enhancement from prior mass loss from the system. The mass of a nova shell is about $10^{-4} M_{\odot}$ (see Paper I). It then requires a maximum of 350 years for the shell to sweep up its own mass in interstellar material. Perhaps the similarity in the spectra occurs because CK Vul 1670 is old enough (unlike the other novae studied here) that it has swept up more than its ejected mass in interstellar material. The emission may be from shock-heated gas at the interface between the expanding shell and the interstellar medium, rather than from the standard 
TABLE 3

Nova Luminosities AND Light Curves

\begin{tabular}{|c|c|c|c|c|c|c|c|c|}
\hline Object & $\begin{array}{c}V_{\text {exp }} \\
\left(\mathrm{km} \mathrm{s}^{-1}\right)\end{array}$ & $\begin{array}{c}\text { Source of } \\
V_{\exp }\end{array}$ & $\begin{array}{c}A_{V} \\
\text { (mag) }\end{array}$ & $\begin{array}{c}m_{V}(\mathrm{max}) \\
(\mathrm{mag})\end{array}$ & $\begin{array}{c}t_{2} \\
\text { (days) }\end{array}$ & $\begin{array}{l}\text { Light Curve } \\
\text { Source }\end{array}$ & $\begin{array}{c}M_{V}(\max ) \\
(\mathrm{mag})\end{array}$ & $\begin{array}{c}M_{V}(15) \\
\text { (mag) }\end{array}$ \\
\hline \multicolumn{9}{|c|}{ Novae of Paper II } \\
\hline v1229 Aql 1970 ..... & 575 & 1 & $1.2 \pm 0.5$ & 6.5 & 18 & 7 & -6.6 & -4.8 \\
\hline v500 Aql $1943 \ldots \ldots .$. & 1380 & 2 & $3.0 \pm 1.5$ & $6.5:$ & 20: & 8 & -10.35 & -8.85 \\
\hline *v1500 Cyg $1975 \ldots \ldots$ & 1180 & 1 & $\overline{1.2}$ & 1.85 & 2.4 & 9 & -0.95 & -5.1 \\
\hline *v446 Her $1960 \ldots . .$. & 1235 & 3 & 0.8 & 2.75 & 5 & 7 & -8.7 & -5.55 \\
\hline *v533 Her $1963 \ldots \ldots$ & 1050 & 4 & 0.6 & 3.5 & 26 & 10 & -7.7 & -6.6 \\
\hline DK Lac $1950 \ldots \ldots \ldots$ & 1075 & 5 & 1.4 & 5.0 & 19 & 5 & -9.35 & -7.35 \\
\hline XX Tau $1927 \ldots \ldots \ldots$ & 650: & 1 & $1.3_{-0.7}^{+0.2}$ & 6.0 & 24 & 11 & -8.05 & -6.75 \\
\hline RW UMi $1956 \ldots \ldots$. & 950: & 1 & 0.1 & $\leq 6.0$ & 200: & 12 & $\leq-7.85$ & $\ldots$ \\
\hline *LV Vul $1968 \ldots \ldots \ldots$ & 860 & 6 & 1.2 & 4.5 & 21 & 6 & -6.75 & -5.25 \\
\hline \multicolumn{9}{|c|}{ Novae Discussed in Paper I } \\
\hline *v603 Aql 1918. & $\ldots$ & $\ldots$ & 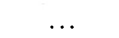 & $\ldots$ & 4 & $\ldots$ & -9.15 & -5.35 \\
\hline T Aur $1891 \ldots \ldots \ldots$ & $\ldots$ & $\ldots$ & $1.2 \pm 0.5$ & $\ldots$ & 80 & $\ldots$ & -7.4 & -7.0 \\
\hline v476 Cyg $1920 \ldots \ldots$. & $\ldots$ & $\ldots$ & $\ldots$ & $\ldots$ & 7 & $\ldots$ & -9.95 & -6.85 \\
\hline *HR Del $1967 \ldots \ldots \ldots$ & $\ldots$ & $\cdots$ & $\ldots$ & 4.6 & $>150$ & $\ldots$ & -5.25 & -5.25 \\
\hline *DQ Her 1934 ........ & $\ldots$ & $\ldots$ & $\ldots$ & $\ldots$ & 67 & $\ldots$ & -6.2 & -5.2 \\
\hline *CP Lac 1936 ........ & $\ldots$ & $\ldots$ & $\ldots$ & $\ldots$ & 5 & $\ldots$ & -9.35 & -5.95 \\
\hline BT Mon $1939 \ldots \ldots \ldots$ & $\ldots$ & $\ldots$ & $\ldots$ & $\ldots$ & $\ldots$ & $\ldots$ & $\ldots$ & $\ldots$ \\
\hline${ }^{*}$ GK Per $1901 \ldots \ldots \ldots$ & $\ldots$ & $\ldots$ & $\ldots$ & $\ldots$ & 6 & $\ldots$ & -8.55 & -5.75 \\
\hline *RR Pic $1925 \ldots \ldots \ldots \ldots$ & $\ldots$ & $\ldots$ & $\ldots$ & $\ldots$ & 80 & $\ldots$ & -7.3 & -6.0 \\
\hline *CP Pup $1942 \ldots \ldots \ldots$ & $\ldots$ & $\ldots$ & $\ldots$ & $\ldots$ & 5 & $\ldots$ & -9.55 & -5.55 \\
\hline T Sco $1860 \ldots \ldots \ldots$ & $\ldots$ & $\ldots$ & 0.6 & 7.0 & 9 & 13 & -8.9 & -6.5 \\
\hline FH Ser $1970 \ldots \ldots \ldots$ & $\ldots$ & $\ldots$ & $\ldots$ & $\ldots$ & 42 & $\ldots$ & -7.55 & -6.55 \\
\hline
\end{tabular}

NoTE.-See text, $\S$ IV $b$ for explanation of asterisks. Sources of data in cols. (3) and (7) are as follows: (1) $V_{\text {exp }}$ from double spectrograph observations at current epoch; (2) Sanford 1943; (3) $V_{\text {exp }}$ from my measurements of spectra from plate vault of Mount Wilson and Las Campanas Observatories; (4) Baschek 1964; (5) Larsson-Leander 1953, 1954; (6) Hutchings 1970; (7) AAVSO (Mattei 1984); (8) Gaposchkin 1943; (9) Young et al. 1976; (10) Chincarini 1964; (11) Cannon 1928; (12) Kukarkin 1963, Ahnert 1963; (13) Sawyer 1938.

mechanisms prevailing in the low-density nova shells themselves. (See Shara, Moffat, and Webbink 1984 for a different interpretation.)

\section{NOVAE AS DISTANCE INDICATORS}

a) The Distances and Luminosities of the Galactic Novae

Distances to individual novae can be obtained directly from the expansion velocities of Table 3 and current shell sizes of Table 2 . I require in addition light curves near maximum light and determinations of interstellar absorption toward each object to derive the luminosities of the novae. The apparent $V$-magnitude at maximum light for each object is adopted from the sources listed in Table 3. I am grateful to the AAVSO for unpublished light curves provided by the Director, Janet A. Mattei (1984, private communication).

The interstellar absorption at $V$ was calculated where possible from the interstellar lines listed in Table 4 using the same

TABLE 4

$W_{\lambda}$ FOR INTERSTELLAR LINES

\begin{tabular}{|c|c|c|c|c|}
\hline \multirow[b]{2}{*}{ OBJECT } & \multicolumn{2}{|c|}{$\mathrm{Ca}$ II } & \multicolumn{2}{|c|}{$\mathrm{Na}$ I } \\
\hline & $\begin{array}{c}3933 \AA \\
(\mathrm{m} \AA)\end{array}$ & $\begin{array}{c}3968 \AA \\
(\mathrm{m} \AA)\end{array}$ & $\begin{array}{c}5889 \AA \\
(\mathrm{m} \AA)\end{array}$ & $\begin{array}{c}5895 \AA \\
(\mathrm{m} \AA)\end{array}$ \\
\hline T Aur 1981 & & $\ldots$ & 900 & 560 \\
\hline v446 Her $1960 \ldots \ldots \ldots$ & 375 & .. & 1300 & 650 \\
\hline v533 Her $1963 \ldots \ldots \ldots$ & $180^{\mathrm{a}}$ & & 660 & 400 \\
\hline DK Lac $1950 \ldots \ldots \ldots$ & $450^{\mathrm{b}}$ & $230^{\mathrm{b}}$ & 1100 & 825 \\
\hline LV Vul $1968 \ldots \ldots \ldots \ldots$ & 295 & $\ldots$ & 1100 & 785 \\
\hline
\end{tabular}

a Agrees with that of Batten, Richardson, and Wright 1963.

b From Larsson-Leander 1954. constants as in Paper I. The $E(B-V)$-distance map of the line of sight to v1500 Cyg 1975 of Young et al. (1976) was used to obtain $A_{V}$ for that object. No interstellar lines could be measured for XX Tau 1927 or RW UMi 1956, as they are now very faint, and no such measurements could be located in the literature. However, I used the $A_{V}$ determined from the total $\mathrm{H}$ I column density toward galaxies within $10^{\prime}$ of each nova given by Burstein and Heiles (1984). This should provide an upper limit to $A_{V}$ for the two novae, which procedure is quite acceptable for RW UMi 1956, since the absorption is very low, and somewhat less so for XX Tau 1927.

No indication of the interstellar absorption could be conjured up for v1229 Aql 1970 or v500 Aql 1943. I therefore adopted $A_{V}=0.5( \pm 0.25) \mathrm{mag} \mathrm{kpc}^{-1}$ as a rough guess for these two objects from my magnitude-limited sample of novae. The latest statistical survey based on UV absorption lines (Shull and van Steenberg 1984) gives a mean value for $A_{V}$ of $0.84 \mathrm{mag} \mathrm{kpc}^{-1}$. A reddening-distance map for these two fields should be constructed as soon as possible. The adopted reddenings are listed in the fourth column of Table 3.

The absolute $V$-magnitudes at maximum light for the novae of Paper I are also included in Table 3. Two changes were made in the data for these novae. The reddening of T Aur 1891 has been redetermined using the new measurements of the interstellar lines. In addition, a $0.2 \mathrm{mag}$ typographical error in $V$ at maximum light of HR Del 1967 from the light curve of Barnes and Evans (1970) has been corrected.

\section{b) The Absolute Magnitude-Rate of Decline Relationship}

All the data which are necessary to derive the absolute magnitude-rate of decline relationship for galactic novae are now assembled. The sample consists of the 10 novae whose 


\section{CK Vul 1670}
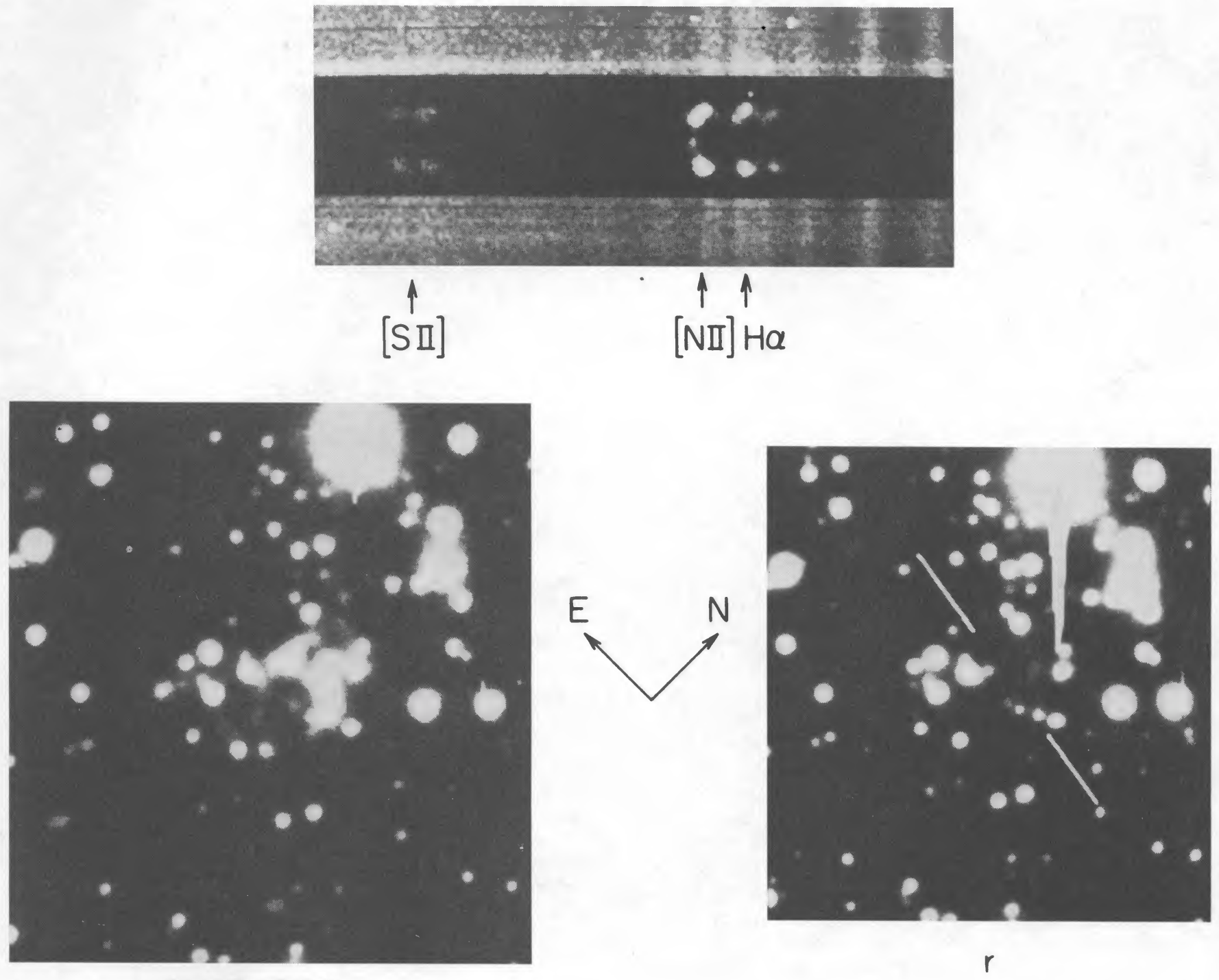

$6570 / 30 A$

FIG. 6.-The sky-subtracted spectrum of the filaments around CK Vul 1670 is shown, as are sections $84^{\prime \prime} \times 84^{\prime \prime}$ of PFUEI images through an $r$-filter and a $30 \AA$ wide $\mathrm{H} \alpha+[\mathrm{N}$ II $]$ filter. The orientation of the spectrograph slit is indicated on the $r$ photograph.

shells were spatially resolved in Paper I and here, the nova T Sco 1860 in the globular cluster M80, the eight shells listed in McLaughlin's (1960, compilation, HR Del 1967 resolved by Kohoutek (1981), and BT Mon 1939 resolved by Marsh, Wade, and Oke (1983). Two of the novae, BT Mon 1939 and RW UMi 1956, have light curves near maximum light which are so poorly observed that the novae cannot be used. (RW UMi 1956 was not even discovered until 1962!)

The parameter I use to characterize the light curve is the time $\left(t_{2}\right)$ in days during which a 2 mag decline from maximum light occurs. Although $t_{3}$ (the time for a $3 \mathrm{mag}$ decline) might, based on the theoretical models of novae (Shara 1981a, b), give a relationship with a smaller dispersion, it will be harder to determine $t_{3}$ for extragalactic novae beyond M31. The $t_{2}$ values adopted from the light curves referenced in column (7) of Table 3 are listed in column (6) of the table. The maximum uncertainty in $t_{2}$ is the larger of 1 day or $10 \%$. The derived absolute $V$-magnitudes at maximum light, $M_{V}(\max )$ (which are corrected for interstellar absorption), are given in column (8) Figure 7 plots $M_{V}(\max )$ against $t_{2}$ for the entire sample of 19 novae. Error bars for the four novae with extremely uncertain reddenings are shown. The 11 novae with $A_{V}<1.5 \mathrm{mag}$, distance $<1.5 \mathrm{kpc}$, and date of outburst later than 1900 (to ensure an accurate apparent magnitude at maximum light) should have the most accurately determined maximum luminosities; they are referred to subsequently as the high-quality sample. The points representing this group are circled in the figure, and its members are indicated by an asterisk preceding the nova name in Table 3 . The least squares fit for these 11 points is

$$
M_{V}(\max )=-10.66( \pm 0.33)+2.31( \pm 0.26) \log t_{2},
$$

where $\log t_{2}$, which has significantly smaller errors than $M_{V}(\max )$, has been used as the independent variable. This fit is indicated by a solid line in Figure 7.

The deviations in $M_{V}(\max )$ of the high-quality sample of novae from the fit have a dispersion of 0.52 mag. This may be 


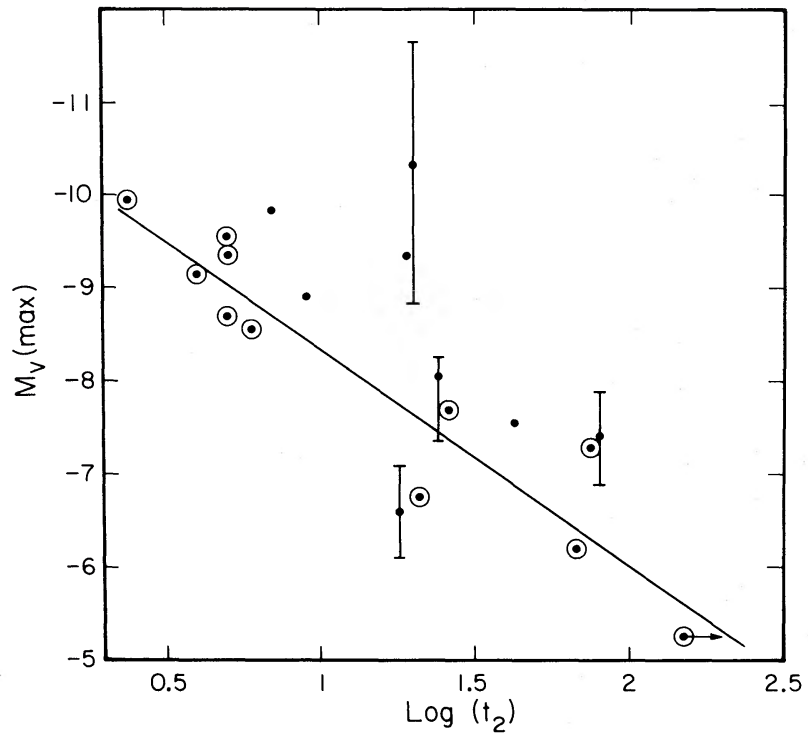

FIG. 7.-The absolute $V$-magnitude-rate of decline relationship for novae with well-determined distances. The solid line is the least squares fit for the high-quality sample (circled points). Error bars are shown where the uncertainty in interstellar absorption is particularly large.

the result of an intrinsic dispersion in the luminosities of nova outbursts or of problems with the observational parameters. I shall return to this point later. The deviations of the nine lower quality points from the mean curve are significantly larger, but can be reasonably explained as problems in the absorption, errors in the shell radii, etc.

\section{c) Using the Theory as a Guide}

The theory of nova light curves, as described by Shara $(1981 a, b)$ and references therein, is now well developed, and specific predictions can be made. The theoretical luminosityrate of decline relationship, using $B$-magnitudes and $t_{3}$ instead of $t_{2}$, is

$$
\begin{aligned}
M_{B}= & -9.5+1.57 \log \left(t_{3}\right)-2.5 \log \alpha+1.79 \log \left(\frac{L_{\mathrm{WD}}}{10^{-2} L_{\odot}}\right) \\
& -5.72 \log \left(\frac{100 R_{\odot}}{R_{\text {phot }}}\right)+2.86 \log \left(\frac{\rho_{\text {phot }}}{3.5 \times 10^{-13}}\right)
\end{aligned}
$$

(Shara $1981 b$, eq. [18]), where $\alpha$ is the ratio of luminosity at outburst to the Eddington luminosity.

Buscombe and de Vaucouleurs (1955) showed that all novae reach about the same magnitude at 15 days after maximum light, while Shara (1981a) predicted that the minimum spread in $M_{B}$ occurs 18 days past maximum light. Pfau (1976) used 11 galactic and 27 extragalactic novae to find $M_{B}(15)=-5.74$ \pm 0.60 . The same light curves as referenced earlier were used to construct $M_{V}(15)$ for the sample of 19 novae, and the results are illustrated in Figure 8. The high-quality sample is distinguished by points enclosed in a circle, as in the earlier figure. The mean value of $M_{V}(15)$ for this sample is $-5.60( \pm 0.14)$ mag, with a dispersion about the mean of 0.45 mag. Values of $M_{V}(15)$ for the lower quality novae scatter widely about this value.

I now assume that the theory is correct, and that $M_{V}(15)$ is in fact a constant. The mean of the observed values of $M_{V}(15)$ of the high-quality sample, -5.60 , is adopted as this constant for all novae. Then the difference between each individual value of
$M_{V}(15)$ derived from the observational data and $-5.60 \mathrm{mag}$ reflects errors in the observational parameters (shell size, absorption, apparent magnitude at maximum light, or expansion velocity). I then use this correction factor to adjust the individual values of $M_{V}(\max )$ deduced from the observations. All errors except those in the shape of the light curve (i.e., in the value of $t_{2}$ ) are then theoretically removed. The resulting $M_{V}(\max$, corr $)-t_{2}$ relationship is shown in the final figure. The relationship has a significantly smaller dispersion than does that of Figure 7. All the novae, including those with lower quality data, were used to determine a least squares fit,

$$
M_{V}(\max , \text { corr })=-10.70( \pm 0.30)+2.41( \pm 0.23) \log t_{2} \text {. }
$$

Note that this fit is within $0.1 \mathrm{mag}$, over the region of interest in $\log t_{2}$, of that of the high-quality sample as defined purely from the observational data. The theoretical relationship (eq. [2]) is linear, with a dispersion about the linear relationship from the last four terms. The constants are somewhat different from the observed values. If I represent $t_{3}$ as $A\left(1.5 t_{2}\right)$, where $A$ must be between 1 and 2, then for $A=1.5$, the theoretical relationship is

$$
M_{B}=-9.15-2.5\langle\log \alpha\rangle+1.57 \log t_{2},
$$

which for $\alpha=1.65$ has the same value as does equation (3) at $t_{2}=10$ days, assuming $B-V$ at maximum light for novae is $0.15 \mathrm{mag}$.

The dispersion of $M_{V}(\max$, corr) for the total sample about the least squares fit in Figure 9 is $0.47 \mathrm{mag}$. This is about the same as that of $M_{V}(\max )$ for the high-quality sample about the least squares fit of Figure 7, and as that of $M_{V}(15)$ of the high-quality sample about the value $-5.60 \mathrm{mag}$. This dispersion thus reflects the intrinsic dispersion in nova properties for a given rate of decline. The third through fifth terms of the theoretically derived $M_{B}(\max )-\log t_{2}$ relationship (eq. [2]) are those that contribute to the expected dispersion, which we have derived from the observational data as $0.47 \mathrm{mag}$. The dispersion calculated purely from the theoretical light curves is unknown (see the discussion in Shara $1981 b$ ) but is at least 0.3 mag.

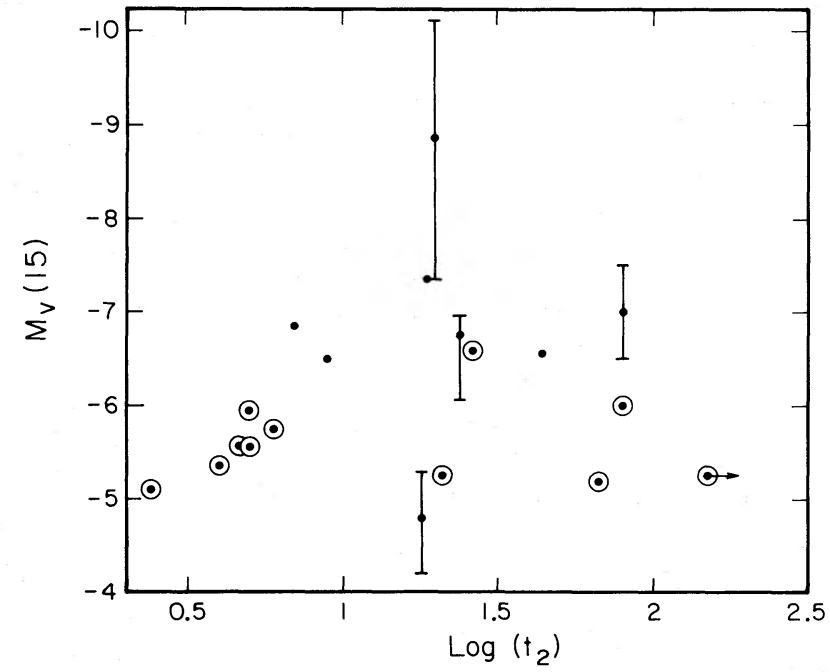

FIG. 8.-The relationship between $M_{V} 15$ days after maximum light and $\log t_{2}$ for galactic novae with well-determined distances. The high-quality sample is indicated as in Fig. 7, and error bars are shown for the novae with particularly uncertain $A_{V}$ values. 


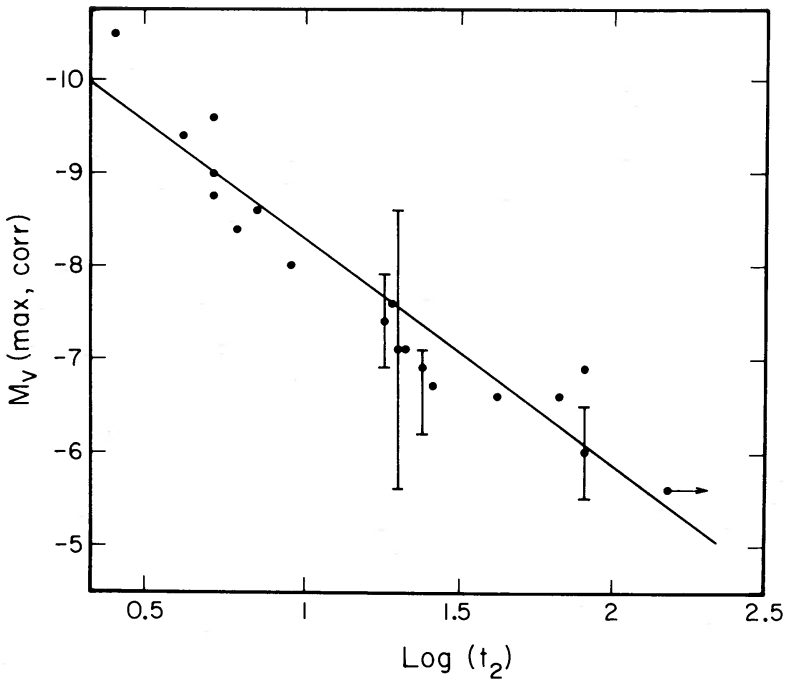

FIG. 9. $-M_{V}(15)$ is assumed to be constant at $-5.60 \mathrm{mag}$, and a corrected value of $M_{V}(\max )$ is derived and shown as a function of $\log t_{2}$. The least squares fit using all the points is indicated by a solid line.

\section{d) The M31 Novae}

The only large sample of extragalactic novae is that of M31. The original data of Arp (1956) can be supplemented by the novae found during the long-term monitoring of the Asiago group. Rosino (1964) reveiewed the data available as of 1963; at that time at least 40 novae in M31 had light curves accurate enough to determine magnitudes at maximum and rates of decline. In his review, Rosino gives the mean $M_{B}(\max )$-rate of decline relationship for the M31 novae. This shows more curvature than the galactic relationship. At the low-luminosity end $[B(\max )>17.5]$, the observational data are marginal, and the slope of the relationship is not reliable. At the highluminosity end ( $t_{2}$ less than 10 days), it is likely that maximum light of many of the novae was missed because of gaps in the temporal coverage. This would explain the apparent curvature below the linear relationship there. The slope in the intermediate region ( $t_{2}$ between 10 and 30 days), where it is best determined, is 2.5, which is identical with the slope of the $M_{V}(\max )-\log t_{2}$ relationship for the galactic novae (2.41). Using this interval in $t_{2}$, one finds that the distance modulus $(m-M)_{0}+A_{B}(\mathrm{M} 31)$ to $\mathrm{M} 31$ is $24.35( \pm 0.20) \mathrm{mag}$. The dominant uncertainty is that of the adopted value of $M_{V}(15)$ for the galactic novae, which is 0.14 mag.

This distance modulus is 0.15 mag smaller than that obtained by van den Bergh (1975) from the same data on the M31 novae, due in part to the new calibration of the luminosities of galactic novae and in part to the relatively low weight I assigned to the brightest M31 novae, where I believe the maxima may have been missed. The new M31 distance is about 0.3 mag smaller than that derived from Cepheids, but this may reflect an inadequate appreciation of the larger mean interstellar absorption within M31 suffered by Cepheids.

\section{SUMMARY}

The attempt to use novae as distance indicators for galaxies requires a galactic calibration using objects with welldetermined distances. The best such candidates are the novae whose shells have been spatially resolved, of which nine were known prior to Paper I. The Palomar search for more spatially resolved nova shells utilized highly accurate digital photo- graphs, through a narrow $\mathrm{H} \alpha$ filter with $\mathrm{CCD}$ detectors, of historical novae with known positions. Two of the older galactic novae, whose locations were poorly known, have been recovered. The search for extended shells involved comparing the radial profile of the image of the postnova star to that of the mean of several nearby stars (i.e., point sources). The radial profiles of the novae were modeled as sums of point sources and extended spherical shells. The influence of the filling factor of the shell and of the filter cutoff has been considered, but only the latter is of importance.

The Palomar search for more spatially resolved nova shells has led to the discovery of 10 such objects, bringing the number of galactic novae with well-determined distances to 21 . Of these, two have light curves that were poorly observed near maximum light and hence cannot be used as distance indicators. The search for more spatially resolved nova shells in our galaxy has now covered most of the novae that were brighter than 6.6 mag at maximum light and that can be reached from the northern hemisphere. Future efforts will be concentrated on the few missing northern objects and the southern novae; the novae of the early 1970s will also be checked again in 5 years. It is fruitless to pursue further the fainter novae, since they are bound to be more distant than those discussed here, and the uncertainties in the substantial interstellar absorption to them become so large that the determination of the parameters of the mean relationships will not be improved.

The spectroscopic observations discussed in § III were taken with a two-dimensional detector during nights of excellent seeing, so that the contribution of the shell and the point source could be separated. In many cases, the dominant contribution to the emission lines comes from the accretion disk (i.e., the point source) rather than the shell. It is thus critical to use expansion velocities measured from the widths of the emission lines from observations during the late stages of decline when the shell emission is still dominant, rather than wait until more than 10 years have passed since the outburst, or to use only observations taken when the shell has expanded sufficiently to become spatially resolved from the point source. The second option is in practice extremely difficult, as by then the shell is very faint. A similar problem occurs for the interstellar absorption, which can be determined from the interstellar absorption doublets of $\mathrm{Ca}$ II and $\mathrm{Na}$. These are also more easily measured accurately close to maximum light when the nova is bright.

The final sample of 19 galactic novae with known distances is divided into 11 objects (the high-quality sample) whose distances are the most accurately determined and the remaining eight novae. I find the luminosity-rate of decline relationship given by equation (1) of $\S \mathrm{IV} b$ for the high-quality sample. The standard deviation in $M_{V}(\max )$ of the 11 points about the mean line is $0.52 \mathrm{mag}$, while, as expected, the lower quality data scatter more widely around the line. The mean value of $M_{V} 15$ days past maximum light is -5.60 (with a standard deviation about the mean of $0.45 \mathrm{mag}$ ) for the high-quality sample.

If I use the well-developed theory of nova outbursts (Shara $1981 a, b)$ as a guide, $M_{V} 15$ days past outburst should be approximately constant for novae of all speeds. Figure 8 shows that this theoretical prediction is at least roughly obeyed. I adopt the mean of the observed values of $M_{V}(15)$ for the highquality sample of novae as this constant value of $M_{V}(15)$. I thus derive a correction factor for each individual object, to produce a luminosity-rate of decline relationship for the entire sample of 19 novae given by equation (3) of $\S \mathrm{IV} c$. The scatter of points 
about this line is small and is the same for the high-quality and lower quality samples. Thus the observational data obey the theoretical predictions of a linear $M_{V}(\max )-\log t_{2}$ relationship, although the constants are slightly different from those predicted from first principles. The observational data, combined with the theory, indicate that the standard deviation in nova outburst properties arising from variations in the outbursts themselves, not from observational errors of any kind, for a given rate of decline is $0.45 \mathrm{mag}$. This is the fundamental limiting accuracy of an individual nova as a distance indicator.

The $M_{B}(\max )-\log t_{2}$ relationship for the novae in $\mathrm{M} 31$, over the interval in $t_{2}$ where it is best determined, is identical in slope with that of the galactic novae. A distance modulus to M31 of $(m-M)_{0}+A_{B}(\mathrm{M} 31)=24.35$ is obtained.

A brief discussion of the peculiar spectrum of the remnant of CK Vul 1670 is given. This object spectroscopically resembles a supernova remnant, but is in fact that of a nova.

I am very grateful to Keith Shortridge, who wrote the software for the Caltech image-processing programs. Diana Foss and Gaston Araya helped with the observations, and Jeremy Mould contributed one spectrum. This research was supported by NSF grant AST 82-12270. I am grateful to the Aspen Institute for Physics for hospitality in 1984 June.

Ahnert, P. 1963, Inf. Bull. Var. Stars, No. 19.

Arp, H. C. 1956, A.J., 61, 15

Barnes, T. G., and Evans, N. R. 1970, Pub. A.S.P., 82, 889.

Baschek, B. 1964, Pub. A.S.P. 76, 22

Batten, A. H., Richardson, E. H., and Wright, K. O. 1963, A.J., 68, 272.

Binette, L. Dopita, M. A., D'Odorico, S., and Benvenuti, P. 1982, Astr. Ap., $115,315$.

Burstein, D., and Heiles, C. 1984, Ap. J. Suppl., 54, 33.

Buscombe, W., and de Vaucouleurs, G. 1955, Observatory, 75, 170.

Cannon, A. J. 1928, Harvard Bull., No. 856, p. 12.

Chincarini, G. 1964, Pub. A.S.P., 76, 289.

Cohen, J. G., and Rosenthal, A. J. 1983, Ap. J., 268, 689 (Paper I).

Gaposchkin, S. 1943, Harvard Bull., No. 917.

Gunn, J. E et al 1984, Bull. A AS, 16,447.

Gunn, J. E., and Westphal, J. A. 1981, in Solid State Imagers for Astronomy, ed.

J. C. Geary and D. W. Latham (Bellingham: SPIE), p. 16.

Hutchings, J. B. 1970, Pub. A.S.P., 82, 603 .

Kohoutek, L. 1981, M N R A S 196,87P.

Kukarkin, B. V. 1963, Inf. Bull. Var. Stars, No. 18

Larsson-Leander, G. 1953, Stockholm Obs. Ann., 17, No. 8. 1954, Stockholm Obs. Ann., 18, No. 4.

\section{REFERENCES}

McLaughlin, D. B. 1960, in Stars and Stellar Systems, Vol. 6, Stellar Atmospheres, ed. J. L. Greenstein (Chicago: University of Chicago Press), p. 585

Oke, J. B., and Gunn, J. E. 1982, Pub. A.S.P., 94, 586.

Payne-Gaposchkin, C. 1957, The Galactic Novae (New York: Dover)

. 1977, in Novae and Related Stars, ed. M. Friedjung (Boston: Reidel), p. 3 .

Pfau, W. 1976, Astr. Ap., 50, 113

Rosino, L. 1964, Ann. d'Ap., 27, 498.

Sanford, R. F. 1943, Pub. A.S.P., 55, 284.

Sawyer, H. B. 1938, J.R.A.S. Canada, 32, 69

Schmidt, T. 1957, Zs. Ap., 41, 182.

Shara, M. M. 1981a, Ap. J., 243, 268.

. $1981 b, A p . J ., 243,926$.

Shara, M. M., and Moffat, A. F. J. 1982, Ap. J.(Letters), 258, L41.

Shara, M. M., Moffat, A. F. J., and Webbink, R. F. 1984, preprint.

Shectman, S. 1978, Annual Rept. Dir. Hale Obs., p. 200.

Shull, J. M., and van Steenberg, M. E. 1984, preprint.

Thuan, T. X., and Gunn, J. E. 1976, Pub. A.S.P., 88, 543.

van den Bergh, S. 1975, in Stars and Stellar Systems, Vol. 9, Galaxies and the Universe, ed. A. Sandage, M. Sandage, and J. Kristian (Chicago: University of Chicago Press), p. 509.

Young, P. J., Corwin, Jr., H. G., Bryan J., and de Vaucouleurs, G. 1976, Ap. J., 209, 882 .

JUDITH G. COHEN : 105-24, California Institute of Technology, Pasadena, CA 91125 